\title{
La enseñanza comunicativa del inglés en el ciclo diversificado en Costa Rica: imágenes de algunas realidades 1
}

\author{
( ,,(dfina Domian Sánchez2, Hilda Fonseca Solórzand \\ lú Ji ío Lara Jiménez4, Sonia Rodríguez Salaza,s \\ I Ir IIV1'rc;idad Nacional, Costa Rica
}

RESUMEN

Análisis sucinto de una investigación de campo efectuada con estudiantes de IO" Y 110 de colegios urbanos y rurales, públicos y privados, de Heredia. La investigación explora las metodologías empleadas por el profesorado y la aplicación del enfoque de la enseñanza comunicati va de la lengua y su relación con el Examen de bachillerato.

\begin{abstract}
A field study was carried out with tenth and eleventh grade students in private and public, urban and rural high schools in Heredia. The research focused on exploring the methodologies used by the instructors as well as on the implementation of the Communicative Language Teaching Approach and its relationship with the Standardized English Test.

Palabras clave: idioma inglés, proceso de enseñanza y aprendizaje, enseñanza comunicativa, enseñanza del inglés, enfoque comunicativo Keywords: English language, teaching-leaming process, communicative teaching, English Language Teaching, Communicative Approach
\end{abstract}

1 Recibido: 27 de abril 2009; aceptado: 7 de enero 2010. Este estudio FONNA parte del proyecto de investigación Análisis del pročeso enseñanza aprendizaje del inglés en el cicló diversificado su relación con la prueba de bachillerato de la Escuela de Literatura y Ciencias del Lenguaje y la División de Educología del Centro de Investigación y Docencia en Educación (CIDE), ambos de la Universidad Nacional, llevado a cabo en 2009.

2 Escuela de Literatura y Ciencias del Lenguaje. Correo electrónico: cdomian@una.ac.cr

3 CIDE. Correo electrónico: hildafonsol @gmail.com

4 CIDE. Correo electrónico: rlara19@ hotrnail.com

5 Escuela de Literatura y Ciencias del Lenguaje. Correo electrónico: srodri@una.ac.cr 
Introducción

En el presente artículo se analiza la metodología empleada por el profesorado de inglés en el ciclo diversificado de veinticinco co- legios de la provincia de Heredia. Se realiza un estudio comparativo entre lo observado en las clases y los lineamientos del Programa de Estudio del Ministerio de Educación Pública de Costa Rica vigente, desde 2005 , así como la bibliografía que sustenta el programa.

Antecedefttes y planteamieftto del problema

Varios aspectos sobre la enseñanza del inglés motivaron el estudio. Se consideraron los comentarios y las críticas leídas en la prensa escrita nacional, las observaciones de algunos docentes en servicio del ciclo diversificado y la experiencia de las investigado- ras como especialistas en lingüística inglesa, lingüística aplicada a la enseñanza del inglés y pedagogía. Se contó con información de algunos artículos periodísticos tales como «Colegiales se gradúan como 'principiantes' en inglés»6, «País enfrenta déficit de 7 .OO tra- bajadores bilingües» 7 , «Educadores desconocen métodos para ense- ñar inglés»8 y la opinión de Osvaldo Muñoz en el «Arte de hablar inglés»9. De estos documentos se concluye que: 
- Después de Matemática, el inglés es la segunda materia en la que fracasan los estudiantes en el examen de bachillerato; en el 2006, un 16\% reprobó este examen.

- El inglés que se imparte en el colegio no reúne los estánda- res que se persiguen con el Programa Costa Rica Multilingüe

6 Jairo Villegas, "Colegiales se gradúan como principiantes en inglés», La Nación 8 mayo 2009: 9A.

7 Jose Carranza, "País enfrenta déficit de 7.000 trabajadores bilingües ", La Nación I abril 2007: 4A. para

8 Jairo Villegas, «Educadores desconocen métodos 2009: 5A. 23

9 Osvaldo Muñoz, «El arte de hablar inglés», La Nación

mayo 2009: 33A. 
(Plan Nacional de Desarrollo 2006-20 1010) lo que implica que los estudiantes se gradúan sin dominar al menos las cuatro des- trezas básicas del idioma, a saber: la expresión oral y escrita y la comprensión auditiva y de lectura.

- La gramática y la traducción imperan como metodología de enseñanza .

Por otra parte, René Quesadall afirma que existe «la posible presión en los educadores para que enseñen para el examen». Esto contrasta con lo fijado en la nueva política del sigloxxı, que procura

«el fomento del aprendizaje de una lengua extranjera que permita aumentar el intercambio con otras culturas y, a partir de allí, entender mejor la propia» ${ }^{12}$.

Con base en los referentes anteriores, si la enseñanza del inglés en Costa Rica se ha caracterizado por ser tradicional , centrada en do- centes y libros de texto, fundamentada en un método gramatical que prioriza el conocimiento estructural y la memorización de listados de palabras, es necesario investigar las posibilidades con que cuenta profesorado para enseñar $-y$ el estudiantado de aprender- el idio- ma a través de un enfoque más comunicativo que le permita superar las situaciones antes identificadas. Es pertinente investigar cómo se restringe o favorece la labor del docente costarricense de inglés para enseñar dentro del enfoque comunicativo, a pesar de que en 14 
la prue- ba de bachillerato se evalúa únicamente un área de desempeño: la comprensión de lectura.

$10 \mathrm{C} \Rightarrow \boldsymbol{r}, 4 \mathrm{~A}$.

11 René Quesada-Inees, Washback Overrides the Curriculum. Tesis doctoral (Chieago: Universily uf Chieago, 2001), 165.

12 Ministerio de Educación Plibliea, Programas de estudio Inglés Educaci6n Diversificada(Sapse!: MEP,2005). 
Referentes teóricos

Una mejor comprensión del proceso de la adquisición de se- gundas lenguas, el surgimiento del inglés como idioma global, el desarrollo científico-tecnológico, la globalización como fenómeno social y el rápido desarrollo de las tecnologías de la información y la comunicación requieren que el proceso enseñanza y aprendizaje de la lengua se lleve a cabo mediante metodologías y aproximaciones con técnicas más participativas que conduzcan al desarrollo de la comunicación oral fluida y eficaz. Lograr un proceso de enseñanza y el aprendizaje eficiente de una lengua extranjera implica que los estudiantes puedan llegar a utilizar la lengua que se aprende con fines comunicativos reales. El enfoque comunicativo (conocido también como la enseñanza comunicativa de la lengua) permite lograr esta comunicación durante la clase.

Este enfoque se originó hacia 1960 a partir de un movimien- to innovador de lingüistas aplicados británicos como reacción a los modelos que parten de la gramática. Tanto los proponentes de Gran Bretaña como de los Estados Unidos de América lo ven como un enfoque y no un método en que lo más importante es desarro- Ilar una competencia comunicativa y abrir procesos de aprendizaje para la enseñanza de las cuatro habilidades lingüísticas que reco- nozcan que el lenguaje y la comunicación comparten un conjunto común de principios 13 . 
El proceso de enseñanza de lenguas extranjeras basado en el enfoque comunicativo destaca la necesidad de ir más allá de la trans- misión y adquisición de un determinado sistema lingüístico; exige que se aprenda a emplear el idioma con precisión, propiedad y des- treza.

Según esto, dominar una lengua implica un conocimiento de estrategias verbales y no verbales de uso y estar familiarizado con los aspectos culturales y pragmáticos de la misma para comunicarse

13 Jack Richards y Theodore Rodgers, Approaches andMethods in Language Teaching (Nueva York: CUP, 1997). 
dc forma eficaz en contextos imprescindibles e impredecibles en el mundo real. En el enfoque comunicativo la clase es un espacio de l'Omunicación. Las actividades de clase giran alrededor de la rela- ción entre las formas y las funciones del idioma, además deben ser relevantes y servir como punto de apoyo para que el estudiantado interactúe entre sí. Para que las actividades logren estos propósitos se acude a materiales auténticos de quienes procuran aprender la len- gua, para crear situaciones de aprendizaje genuinas y significativas las cuales propicien un ambiente colaborativo y que no sea amenaza- dor. La enseñanza está centrada en el estudiante y en sus necesidades comunicativas. Se le da la oportunidad de compartir y explorar acti- tudes, sentimientos y opiniones, y de concentrarse en su proceso de aprendizaje mediante la comprensión de su propio estilo de aprender y el continuo desarrollo de las destrezas y habilidades necesarias para adquirir una lengua. Los estudiantes tienen más independencia y, por lo tanto, mayor responsabilidad en la toma de decisiones sobre

su aprendizaje. El profesorado tiene la función de guía, consejero, organizador y facilitador. Éste y los programas se relegan a un se- gundo plano y son las necesidades de los estudiantes el escenario sobre el que se monta la actuación de los docentes 14

El enfoque comunicativo en la enseñanza de idiomas parte de que la lengua es comunicación e 18 
interacción. Se traza como objeti- vo el desarrollo de la competencia comunicativa, noción procedente de Noam Chomsky quien se refirió a una competencia lingüística, entendida como la capacidad del oyente/hablante ideal para reco- nocer y producir una infinita cantidad de enunciados a partir de un número finito de reglas en una comunidad lingüística homogé- nea15. Más tarde, Dell Hymes introdujo la noción de competencia comunicativa como la capacidad de actuar verbalmente de modo

14 James F. Lee y Bill Van Patten, $M q_{\mathbf{k}}$ ing Communicative Language Teaching Happen (Boston: McGraw Hill, 2(03) 49-53.

15 Betsy Parish, Teaching Adult ESL. A Practical Introduction (Nueva York: McGraw Hill. 2004) $31-32$. 
eficaz en una comunidad de habla, no solo con enunciados grama- ticalmente correctos sino que también adaptándose al interlocutor, el propósito, la situación, el contexto y el género discursivo. Hay que saber qué decir a quién, cuándo y cómo. Los conceptos se han ido reelaborando con aportaciones de diversos investigadores; uno de los modelos más conocidos es el de Michael Canale y Merril Swain, quienes propusieron tres componentes (competencia gra- matical, disCursiva y estratégica), ampliado a cuatro (competencia sociolingüística) por Canale!6:

- La competencia gramatical: dominio del código lingüístico que incluye el conocimiento sintáctico, léxico y fonológico de la lengua;

- La competencia discursiva: dominio para combinar las formas

gramaticales y significados para lograr un texto coherente y fluido ya sea hablado o escrito;

- La competencia estratégica: dominio de las estrategias de

comunicación verbal y no verbal para resolver problemas comunicativos, que ocurren por

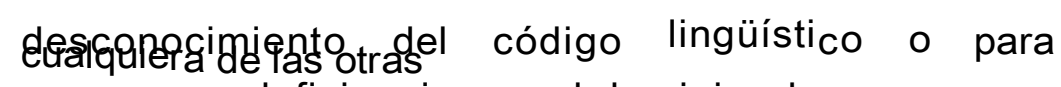
compensar deficiencias en el dominio de

- La competencia sociolingüística: dominio de las normas que regulan el uso adecuado del código 
lingüístico en los distintos contextos sociológicos y de situación comunicativa.

El concepto de competencia comunicativa ha servido de base tanto para el currículo lingüístico de muchos países como para algu- nos documentos comunes de referencia para el aprendizaje y ense- ñanza de las lenguas, y en particular en el Marco Común Europeo de Referencia para las Lenguas!7. Se utiliza desde la década de 1970,

16 Lyle F. Bachean, Fundamental Considerations in Language Testing (Oxford: OUP,2004) 84-100.

17 Consejo de Europa, "Marco Común Europeo de Referencia para las Lenguas», <http://cvc.cervan- tes.es/ensenanzalbi blioteca_ele/marco>,2001, consultado 10 setiembre 2011. 
en el caso de la enseñanza de lenguas extranjeras, y desde 1990 en el raso de la enseñanza y el aprendizaje de las lenguas maternas.

La enseñanza comunicativa de la lengua (en inglés, Commu-

„;'cative Language Teaching) es un enfoque, más que un método, porque en cuanto a la teoría de la lengua y del aprendizaje existe IIn marco teórico consistente; sin embargo, en cuanto al diseño y procedimientos, da para una mayor interpretación y variación indi- vidual que el que permite la mayoría de los métodos. Según Brown, este marco teórico se ha enriquecido con los aportes de Allen y Widdowson (1 975), Halliday (1 970), Savignon (1 971, 1 972), John- son y Morrow, Stern (1978), Wilkins (1 976), Widdowson y muchos otros lingüistas, lo que lo convierte en un marco teórico siempre en evolución, razón por la cual es difícil ofrecer una definición de la enseñanza comunicativa de la lengual8'

En muchos países, incluido Costa Rica, la enseñanza comuni- cativa ha formado parte de los programas de la enseñanza de inglés como lengua extranjera desde 1970. En 2003 bajo el lema «relanza- miento de la educación costarricense» el Ministerio de Educación Pública (MEP) rediseñó los programas de estudio de la educación secundaria dentro de los cuales se encontraban el de inglés como len- gua extranjera para el ciclo diversificado. Según los Programas de Estudio 2005 Inglés Educación Diversificada19, 
el nuevo programa se elaboró con el objetivo de desarrollar la competencia comunica- tiva del estudiantado en la lengua extranjera. Se alude a que el ob- jeto de estudio del inglés es la comunicación oral y escrita y se hará hincapié en las cuatro habilidades: comprensión lectora y auditiva y expresión escrita y oral a las que se les dedicará el mismo tiempo. Además, se considerarán las necesidades y los estilos de aprendizaje para ofrecer al estudiantado oportunidades que le permitan un uso significativo de la lengua. El programa no se refiere a los principios

18 H. Douglas Brown, Principies ofLanguage Learning and Teaching (Nueva York: Pearson, 207) 241-242.

19 MEP, 13. 
de una enseñanza basada en la estructura lingüística de la lengua; por el contrario, se parte de que el enfoque comunicativo será la base de la metodología. En el anexo 2

del documento citado anteriormente se indica lo siguiente:

«Para la elaboración de los programas de inglés general (GE) para la Educación Académica y para ,los cursos de inglés especializado, para la Educación Técnica, Se ha tomado confol)pase filosófica los postulados del enfoque comunicativo para las lenguas extranjeras, los principios de John Munby (citado en MEP en su libro Communicative Syllabus Design Iajs funciones del lenguaje, que hace posible los actos de habla».

No obstante que gran parte de la población costarricense ad- mite la necesidad de aprender inglés Como medio para comunicarse en este mundo globalizado, los métodos pedagógicos tradicionales para la enseñanza de lenguas extranjeras se imponen y limitan el desarrollo de la competencia comunicativo. Además, el profesorado de inglés del ciclo diversificado en Costa Rica tiene como primera responsabilidad que sus estudiantes aprueben el examen de Bachille- rato. Se trata de un examen que no mide las cuatro habilidades, sino solo la comprensión de lectura; razón para que el enfoque comuni- cativo no se implemente de manera consistente ya que el concepto original debe ser modificado para ajustarse a la realidad.

Metodología 
Para esta etapa de la investigación se utilizó el paradigma cua- litativo que Locke, Spirduso y Silverman20 (citado en Creswell), em- plean con el fin de comprender una situación social, evento, papel,

20 James. Creswell. Research Design: Qualitative and Quantitative Approaches. (Londres: SAGE Pub1ications, 1994), 58. 
grupo o interacción en particular. Miles y Huberman21 (citado en Creswell) indican que en lo fundamental, el método cualitativo de investigación es un proceso exploratorio donde quienes investigan logran darle sentido a un fenómeno social al contrastar, comparar, replicar, catalogar y clasificar el objeto de estudio.

Con el apoyo del Instituto de Estudios en Población, de la Uni- versidad Nacional, la muestra se tomó de forma aleatoria incluidos los docentes del ciclo diversificado diurno, de colegios públicos y privados de todos los circuitos de la sede regional del MEP de Here- dia y los dos circuitos de la sede del MEP del cantón de Sarapiquí. Igualmente se consideraron las posibilidades del equipo investiga- dor. Al final, la muestra permitió la selección de treinta y un docentes de la provincia de Heredia, entre veinticinco colegios22

Las observaciones a los docentes se realizaron in situ durante un periodo de nueve meses durante 2009. Se programaron cuatro visitas a cada clase en un mismo horario, de forma semanal. En total se efectuaron 77 observaciones no participativas cada una con un promedio de dos lecciones de 40 minutos. En el estudio se han incluido las impresiones de dos de los sujetos quienes lograron mante- ner una clase comunicativa exitosa y sus perspectivas debían tenerse en cuenta. La negociación de permiso y entrada a las instituciones educativas se inició en 2008, con gestiones ante la Sede Regional de Heredia y la 20 
Sede Regional de Sarapiquí del MEP. En ese año se informó sobre los objetivos principales del proyecto de investigación y el siguiente año se solicitó el permiso correspondiente para visitar a los grupos seleccionados.

21 Creswell, 59.

22 El liceo de Heredia, liceo Manuel Benavides, Colegio Bilingüe de Belén, El Centro Educativo Santa Inés,EColegio María Auxiliadora, Ebnservatorio Castella, El Colegio Claretiano, EI Colegio Aarora, El Taller Pedagógico Montebello, ElColegio Bilingüe Santa Cecilia, El liceo del Roble, IPEC Barva, EI liceo Rodrigo Hemández, EI liceo San José de la Montaña, Saint Nicholas oC Rüe,EColegio Bilingüe del Monte, El CEUNA, EColegio Nueva Generación, El liceo San de Isidro, El üceo Mario Vindas, Elolegio Santa María de Guadalupe, El Colegio Deportivo Santo Domingo,EColegio Vilaseca, Cblegio de Río Frío y El liceoAmbientalista en Horquetas de Sarapiquí. 
Para recolectar y analizar la información, se utilizó un instru- mento que permitió hacer un regi stro de manera focalizada de las técnicas y actividades (ver Anexo); el instrumento está basado en las características del enfoque comunicativo descrito en Principies of Language Learning and Teaching23, de Brown. Terminada la fase de observación en noviembre de 2009 , la tabulación se realizó de forma manual y se analizó cada uno de los comentarios que las investigadoras apuntaron según su respectivo criterio de expertas. En el si- guiente apartado se resumen los aspectos metodológicos observados en cada clase que no coinciden con el enfoque comunicativo y que se aplicaron en la mayoría de las clases observadas.

Resultados

Se ha organizado la información en categorías que se derivan de seis características generales del enfoque comunicativo, vistas como las más representativas a través de las observaciones. Así los resultados se agruparon en: metodología y producción espontánea; uso del idioma español; motivación intrínseca del estudiante; ense- ñanza de distintas variedades del lenguaje; integración de las cuatro destrezas básicas; y evaluación.

Sobre la metodología y producción espontánea

- La organización física del aula no facilita la enseñanza 
comu- nicativa: los estudiantes se ubican en filas y en semicírculo sin ningún objetivo.

- Los estudiantes no muestran su capacidad de comunicación oral en inglés: interactúan en español . Hablan inglés solo cuando el docente se dirige a ellos para formularles preguntas. No se usa el inglés al dar instrucciones, disciplinar, organizar la clase, brindar estrategias de lectura y explicar aspectos gramaticales 
complejos. En ocasiones se traduce al español inmediatamente que se indica la instrucción en inglés.

- Las actividades de lectura con preguntas de comprensión y vocabulario en contexto toman la mayoría del tiempo de las lecciones. Se acentúa la compresión de lectura en la mayoría de las clases observadas.

- Los diálogos se centran en funciones del lenguaje pero son memorizados o leídos, incluidos aquellos que los estudiantes formulan. Se usan también como forma de entender una es- tructura específica o contenido nada más. Los estudiantes pre- paran por escrito lo que van a decir y luego lo verbalizan.

- Hay pocas oportunidades para la comunicación en inglés entre el estudiantado se comuniquen entre sí, y con poca supervisión de parte del profesorado.

- Los contenidos, más que la funcionalidad del lenguaje, pre- dominan en la clase . Se enseña para dominar una estructura; por ejemplo, se pregunta: Did Ivannia come to school today? Las personas presentes saben que 1 vannia vino a clase. Las situaciones de las actividades son ficticias; o sea, la oportuni- dad que se pudiera dar de comunicarse de forma real en clase no existe.

- Se utiliza la lectura de un texto en voz alta para estudiar el sig- nificado del vocabulario nuevo y luego proceder a contestar los ejercicios de comprensión de lectura. 
En muy pocas ocasiones se observó que se utilizara para práctica de pronunciación.

- El profesorado controla las respuestas siempre, lo que no im- pide al estudiantado analizar y experimentar con el idioma. La corrección se realiza dando la respuesta correcta en lugar de buscar el análisis o la introspección.

- El fotocopiado es excesivo, se pierde tiempo si los estudiantes no llegan con las copias. Algunos salen a sacarlas cuando ya ha empezado la clase. 
- Los «calentamientos» y las «lluvias de ideas» son escasos.

- El uso del inglés no se modela de forma adecuada: ${ }^{*}$ Carlitos, you did tt?

- Los ejercicios de completar espacios son los más habituales.

- A veces las unidades se inician diez minutos antes de finalizar la clase.

- Existe el espacio para que los estudiantes expongan sus infor- mes y hagan presentaciones orales; sin embargo, los docentes les permiten leerlo con ausencia de realimentación.

- A pesar de que hay actividades orales, no se tiene clara la fun-

ción o la destreza que se desarrolla con esa actividad.

- Se siguen los contenidos sin una relación con el sistema lingüístico.

Sobre el uso del idioma español

- El estudiantado suele utilizar el español tanto para el manejo de clase como para el contenido; rara vez responden en inglés. Se dan algunas instancias solo cuando cada docente les hace preguntas directas en inglés sobre el contenido.

- Las estructuras más complejas son traducidas del inglés al es- pañol por la mayoría de los docentes y van acompañadas con explicaciones en la lengua materna. Uno de estos ejemplos es la traducción de 
la estructura gramatical: Wouldyou mind.. ?

Sobre la motivación intrínseca de quienes aprenden la lengua

- El trabajo grupal no es una actividad significativa para los es- tudiantes en el proceso de aprendizaje de la lengua extranjera. Se trabaja en grupo solo para responder preguntas de com- prensión de lectura, revisar vocabulario y completar ejerci- cios de gramática. El trabajo se limita a formar grupos, pero no se aprecia una definición del docente en cuanto a los fines comunicativos en este tipo de agrupación ni responsabilidad de aprendizaje comunicativo en la mayoría de los estudiantes. 
En algunos grupos de trabajo se observa la asignación de un tutor o coordinador; sin embargo, no se le responsabiliza clara- mente por el rol asignado.

- El uso del diccionario para la traducción de términos provoca apatía y desinterés. Esto se ilustra con frases tales como «i Otra vez!», «¿Cuántas palabras son hoy?» y «i Qué aburrido!».

- Todos los estudiantes deben cumplir el ritmo de trabajo puesto que no hay en el planeamiento diferenciación entre los distin- tos niveles de los estudiantes y sus estrategias de aprendizaje.

- El MEP prepara la secuencia de unidades por temáticas por lo que en la mayoría de las observaciones no se evidenció que se diera la oportunidad de escoger subtemas de interés particular para el grupo de estudiantes.

Sobre la enseñanZl de distintas variedades del lenguaje

Por los datos disponibles, el docente no aprovecha los recursos disponibles para enseñar los registros y las distintas variedades del lenguaje que aparecen en materiales auténticos, tales como pelícu- las, documentos, periódicos nacionales e internacionales en inglés, revistas y audiovisuales.

Sobre la integración de las cuatro destreZls básicas

En las clases de los docentes que integran las cuatro 
destrezas lingüísticas (mayormente de colegios privados/subvencionados), se observan los siguientes patrones:

- Se presenta en los colegios privados la posibilidad de utilizar re- cursos tecnológicos que potencien las cuatro destrezas tales como sala de proyección, televisor, reproductor de video y de audio.

- La lectura se utiliza como generadora de discusión, investiga- ción y presentaciones orales. 
- El educto (comprehensible input) ofrecido por el docente así como el material audiovisual son utilizados para generar discusión.

- Sin embargo, la media gira alrededor de aquellos instructores que no logran la integración .

- Principalmente se privilegia la comprensión de lectura por lo general seguidos de ejercicios básicos de ubicación de infor- mación; en menos ocasiones, ejercicios más complejos como la relación de ideas y el análisis crítico del contenido.

- La escritura es básica, limitada a completar espacios en con- textos cortos como oraciones y párrafos.

- La expresión oral espontánea se reduce al mínimo.

- La gramática se enseña al final, como forma de revisión, y mu- chas veces tomada del libro.

- Las microhabilidades se enseñan en español ; en su defecto, aquellas para la escucha y comprensión escrita no se dan.

- $\quad$ Algunos ejercicios inician al final de la lección (escucha) y no hay realimentación .

- Se enseña el lenguaje funcional más que los otros componen- tes lingüísticos (el gramatical, el discursivo, el sociolingüístico o estratégico).

- Se enseña el idioma con el fin de superar una prueba de medi- ción. Así fue expresado por algunos docentes a sus estudiantes con el fin de que prestasen 
atención a la lección. Por ejemplo, el docente manifiesta a sus estudiantes: Este vocabulario es importante para el examen .

Sobre la evaluación

La mayor parte de las evaluaciones se ajustan al ordenamiento institucional (pruebas de desempeño escrito y comprensión de lectura) dentro del periodo establecido por el MEP para la rea- lización de pruebas parciales ofinales del trimestre. 
- No se utilizaron escalas o rubros específicos para el registro de la producción oral.

Análisis de los resultados y discusióft

Se ha organizado el análisis de los resultados conforme a seis temas fundamentales: la enseñanza comunicativa del inglés, la utilización de los recursos educativos, los ambientes áulicos, la di versidad de actividades comunicativas, la relación de las metodologías con la prueba de bachillerato y el mérito que algunos do- centes merecen.

Con respecto a la enseñanza comunicativa del inglés

Los docentes tienen imprecisos conocimientos sobre la ense- ñanza comunicativa del inglés. Las diversas técnicas aplicadas mos- traron contradicciones e incongruencias con esos conocimientos. Solo el 38,70\% (12) del profesorado mostraron un desempeño que supera la tesis anterior en cuanto al:

I. uso del inglés para desarrollar actividades como presentar con- tenidos y orientar actividades de práctica y producción, dar instrucciones para la organización de la clase, atender la disci- plina y realimentar el desempeño de cada estudiante;

2. desarrollo de experiencias de aprendizaje que contribuyen a la comunicación que conlleve 
propósitos prácticos;

3. intento de integrar las cuatro habilidades básicas;

4. empleo de modalidades verbales y no verbales (speech arrange- ments) tales como explicaciones $\mathrm{y}$ demostraciones;

5. el fomento de la adquisición de la lengua para comunicarse de fonna espontánea y no la simple transmisión del sistema lingüístico del inglés. 
De estos docentes ( $\left.\begin{array}{ll}1 & 2\end{array}\right)$, dos consideraban motivante aplicar el enfoque comunicativo en sus clases. Manifiestan que se incrementa la participación espontánea de los estudiantes y su autonomía a la hora de producir. Es evidente el avance de los estudiantes puesto que se marca un cambio en la fluidez. Las clases son más activas y los estudiantes se involucran en las actividades comunicativas. Un docente se refiere a la satisfacción del estudiantado cuando logran comunicarse en inglés, a pesar de los errores. Ellos practican el idio- ma al conversar y realizar actividades comunicativas.

Pese a los obstáculos potenciales en el empleo del enfoque co- municativo, las docentes que lo utilizan para la enseñanza del inglés indican que las fortalezas superan las debilidades y los resultados son sobresalientes en la práctica y así se evidencia la eficacia en la competencia comunicativa de los beneficiados.

Por otra parte, el $61,30 \%$ (19) del personal docente evidenció las incongruencias con los principios de la enseñanza comunicativa. Algunas prácticas educativas ilustran esta afirmación: la enseñanza a partir de puntos gramaticales a pesar de que tanto el plan de estudio del MEP como otros textos utilizados exponen los contenidos por ejes temáticos y por funciones del lenguaje; el aprendizaje de las funciones se limita a completar ejercicios en un libro; el desarrollo de actividades orales no es espontáneo, pues se aceptan diálogos leí- dos y 34 
previamente elaborados con pleno control del profesor, muchas veces con ausencia de realimentación ; y la realimentación directa en forma de corrección en el momento inadecuado. Esto interrumpe el discurso del estudiante mientras elabora sus ideas o lo inhibe.

De forma similar, dos profesoras manifestaron que acudieron

al método comunicativo, pero encontraron debilidades en su empleo. Uno de ellos es la indisciplina que se deriva del trabajo con gru- pos de 35 a 45 estudiantes. Además, manifestaron la dificultad de construir una cultura de aula en que los estudiantes trabajen solos sin depender del profesor y en que cada uno de ellos en los grupos 
pl"lueños tenga un papel del cual deba responsabilizarse. Para una dl' las docentes, otra dificultad en la utilización del enfoque comuni- I"iltivo consiste en que existen distintos niveles de conocimiento del Idioma inglés en la misma sección. Esto hace que el profesor busque diferentes estrategias para los niveles intermedios o bien aquellas 'lue se adapten al nivel mayoritario de los estudiantes en grupos con tanta población. Otro de los obstáculos es el tiempo necesario para realizar diversas actividades comunicativas ya que suelen exceder el tiempo de clase para concluirlas correctamente. Muchas de las acti- vidades deben interrumpirse y postergar su seguimiento. Coinciden en que una de las desventajas para utilizar el enfoque comunicativo es enlazar la metodología utilizada con la evaluación propuesta por el MEP ya que se trata de una evaluación en la cual predomina la medición y las pruebas escritas.

Con respecto a la utilización de los recursos educativos

En general, el profesorado realizó un uso limitado de materia- les lingüísticos, lo que llevó a que no se les ofreciese a los estudian- tes una variedad de recursos tecnológicos en sus procesos de apren- dizaje del inglés. El personal docente utiliza materiales auténticos tradicionales como los periódicos y las revistas, o modernos, como cintas, videos o computadoras para facilitar el aprendizaje así como lo propone el método comunicativo; la mayoría limitó su enseñanza a un uso excesivo de documentación 
fotocopiada . De esta manera, las experiencias observadas carecieron de esa oportunidad de ofrecer al estudiantado variedad de contextos lingüísticos y culturales como lo plantea tanto el MEP como el enfoque comunicativo.

Con respecto al uso del espacio

La organización en el aula y en particular la disposición del mobiliario restringió la enseñanza y el aprendizaje comunicativo. En la mayoría de las clases observadas, los pupitres se colocaron 
en filas, lo que no promueve la comunicación entre quienes par- ticipan en una comunidad educativa. En algunos casos, el grupo de estudiantes, a pesar de su colocación en semicírculos, no inte- ractuaba en la lengua meta. Habrá que explorar las razones de la organización mobiliaria de las clases de inglés e indagar si obedece a limitaciones de infraestructura, la necesidad de control, a una cultura pedagógica nacional, a un lineamiento institucional o a la necesidad del personal docente de tener atención o control visual y verbal sobre sus pupilos.

Con respecto a la di'Jlersidtul de actividades comunicativas

Se hizo evidente una limitada diversidad de actividades co- municativas en la mayoría de las clases observadas, además que las lecciones de inglés eran predecibles. Actividades como las presen- taciones orales leídas de los estudiantes, un exceso de actividades de comprensión de lectura, ejercicios para completar y otros que involucran traducción, fueron actividades usuales en los ambientes observados. Según varios exponentes del enfoque comunicativo24, aquellas actividades cuyo objetivo es la comunicación genuina au- nado a la promoción del uso del idioma y la motivación para que los estudiantes interactúen entre sí en inglés, no fueron observadas en los contextos del estudio.

Con respecto a la relación de las metodologías con la prueba de 38 
Se detectó una preocupación del profesorado y del estudiantado

por la práctica de comprensión de lecturas similares a las de las prue- bas de bachillerato; no por la comprensión de lectura. Tanto en las actividades de la clase como en los ejercicios en los exámenes, se notó

24 Diane Larsen-Freeman, Techniques and Principies in Language Teaching (Nueva York: Oxford University Press, 1986 ); William Littlewood, Communicative Language Teaching (Cambridge: Cambridge University Press, 1981); y Jack Richards. "Communicative Language Teaching To- day,.. Cambridge.com 2005. Web. 4 marzo2009. 
1 '1 acento en la exposición del estudiante a las lecturas. Complemen- tariamente a los libros de texto, se usaban folletos en los que practi,'aron con rutina en ciertos horarios o después de algunas activida- eles. Sin embargo, no se puede afirmar que la naturaleza del trabajo $\mathrm{nm}$ las lecturas potenciara diferentes tipos de conocimiento; a saber: nmocimiento, comprensión, aplicación, análisis, síntesis y evalua- l'illn. Antes bien, los ejercicios a los que se sometía los estudiantes esas lecturas se centraban más en la ubicación de información en el texto; esto es, reconocimiento de eventos en las lecturas con los trucos lingüísticos de sinónimos, antónimos y otros que promueven mayormente la localización de datos . Esta dinámica de comprensión de lectura es similar a la que los estudiantes enfrentan en la prueba de Bachillerato, asunto en que se profundizará en otra oportunidad.

Con respecto a los méritos particulares de algunos docentes

Una minoría de docentes maneja con claridad las estrategias y técnicas propuestas en el enfoque comunicativo. La comunicación en la segunda lengua dentro y fuera de la clase, la utilización de distintas modalidades verbales y no verbales (modelar, parafrasear, interrogar, utilizar variantes en la pronunciación, entre otros), la sostenibilidad del uso oral de la segunda lengua por parte del docente en clase, la aceptación del español por parte del estudiante en algunos momentos, la utilización de recursos 40 
como videos, cintas y material auténtico como periódicos que faciliten las imágenes (viñetas) cultu- rales y diferentes variantes lingüísticas, son algunas de los esfuerzos comunicativos observados en una pequeña cantidad de los docentes observados en este estudio. Cabe recordar que este fenómeno fue observado en colegios tanto públicos como privados, y más notable en los segundos. 
Conclusiones

Según los resultados expuestos, el enfoque comunicativo no se aplica a cabalidad en los centros educativos del ciclo diversificado. Aunque en términos generales, esta aseveración se oye con frecuen- cia, el presente análisis es un estudio sistemático que comprueba lo que sucede con las materias de inglés. Existen intentos de aplicar metodologías comunicativas, pero falla el ciclo completo ya que la producción de lenguaje significativo no se da. Más que un notable y medible obstáculo de idioma en una parte del profesorado, la caren- cia mayor está en sus aplicaciones metodológicas.

No obstante, se debe considerar el contexto cultural y las po- líticas educativas dentro de las cuales se da el proceso enseñanza y aprendizaje del inglés en Costa Rica. Al profesorado se le puede ca- pacitar en el uso de nuevas técnicas, formular propuestas, plantearle una serie de actividades participativas para desarrollar la competencia comunicativa en quienes estudien el idioma y para que la labor del profesor tenga más consistencia en la clase y las lecciones motiven al estudiantado a comunicarse en inglés. Sin embargo, no se pueden olvi- dar las limitaciones a las que se enfrenta el personal docente del ciclo diversificado de la mayoría de las instituciones educativas, tales como la falta de materiales y laboratorios de idiomas y de informática, la presión que ejercen los padres de familia, otros docentes y el 
sistema administrativo, la preparación del estudiantado para realizar la prueba de bachillerato en inglés y su situación socio-económica. Otra limita- ción, según el MEJ>25 (Villegas, 13 de mayo de 2009) con respecto a los docentes, es que el $86 \%$ de ellos a mayo de 2009 no cuentan con un buen dominio del inglés; de acuerdo al presente estudio, se percibe

25 Jairo Villegas , «2.000 docentes de inglés a capacitación», La Nación.com, 13 de mayo de 2009. Web. 28 de noviembre de 2009 . 
Inseguridad por parte del profesorado para desarrollar un programa en

"y que se prioriza la comunicación oral fluida y eficaz.

Pese a lo anterior, es plausible que todos los esfuerzos que ha Iniciado el Consejo Nacional de Rectores (CONARE) apunten a for- lalecer y mejorar el aprendizaje del inglés a nivel nacional. Aún que- da camino por recorrer y no hay duda del empeño de los docentes de l'nseñanza bilingüe del país de realizar su trabajo de la mejor manera. 

Anexo: Formulario utilizado en observaciones

Universidad Nacional

Centro de Investigación y Docencia en Educación

División de Educología

Facultad de Filosofía y Letras

Escuela de Literatura y Ciencias del Lenguaje

Análisis del proceso de enseñanza y aprendizaje del inglés en el ciclo diversificado y su relación con la prueba de bachillerato

School:

Observer's Dame:

Teacher's Dame:

Visit Dumber:

Date:

\begin{tabular}{|c|c|c|c|c|}
\hline $\begin{array}{c}\text { Characteristics of Communicative } \\
\text { Language Teaching }\end{array}$ & $\mid \begin{array}{l}\text { Oell1: } \\
\theta \\
11 \\
0\end{array}$ & 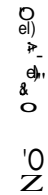 & Comments & \\
\hline $\begin{array}{l}\text { a) Vnrehearsed ianguage perfor- } \\
\text { manee. }\end{array}$ & 1 & 2 & & $-1-1-$ \\
\hline $\begin{array}{l}\text { 'b)- Stud Ints are encouraged to } \\
\text { Ideal with unrehearsed situa- } \\
\text { 'tions underlhe g.uiciance (but } \\
\text { 'nó co trol) oc thefe cher. }\end{array}$ & 1 & 2 & & $-1-1-$ \\
\hline $\begin{array}{l}\text { e) Students are given toolsió ge- } \\
\text { nerate unrehearsed language. }\end{array}$ & 1 & 2 & & $-1-1-$ \\
\hline $\begin{array}{l}\text { d) Students are encouraged to } \\
\text { communicate in English. }\end{array}$ & 1 & 2 & & $-1-1$ \\
\hline $\begin{array}{l}\text { e) Class facilitates lifelong lan } \\
\text { guage learning. }\end{array}$ & 1 & 2 & & $1-1-$ \\
\hline
\end{tabular}




\begin{tabular}{|c|c|c|c|c|}
\hline 1) & Meaning is more important. & I & 2 & $I_{-} I_{-}$ \\
\hline & $\begin{array}{l}\text { Dialogues are centered on } \\
\text { communicative functions and } \\
\text { are not memorized. }\end{array}$ & I & & $-L_{2}$ \\
\hline h) & $\begin{array}{l}\text { Drilling is part of an activity or } \\
\text { situation, not a main component. }\end{array}$ & I & 2 & $-1-1$ \\
\hline & $\begin{array}{l}\text { Comprehensible pronunciation } \\
\text { is encouraged. }\end{array}$ & I & 2 & ${ }_{-} I_{-} I_{-}$ \\
\hline & $\begin{array}{l}\text { Sensible and careful use of } \\
\text { native language is acceptable } \\
\text { when necessary. }\end{array}$ & I & 2 & ${ }_{-} I_{-} I_{-}$ \\
\hline & $\begin{array}{l}\text { Linguistic system of English is } \\
\text { taught through the process of } \\
\text { communication, not by teach- } \\
\text { ing grammar. }\end{array}$ & I & 2 & ${ }_{-} I_{-} I_{-}$ \\
\hline & $\begin{array}{l}\text { Sequence of units is detenni- } \\
\text { ned by consideration of con- } \\
\text { tent, function or meaning that } \\
\text { maintains students' interest. }\end{array}$ & I & 2 & ${ }_{-} I_{-} I_{-}$ \\
\hline & $\begin{array}{l}\text { Intrinsic motivation comes } \\
\text { from an interest in what is be- } \\
\text { ing communicated by the lan- } \\
\text { guage not from an interest in } \\
\text { the grammar of the language. }\end{array}$ & I & 2 & ${ }_{-} I_{-} I_{-}$ \\
\hline n) & $\begin{array}{l}\text { Students are partners in a ro- } \\
\text { operative venture. }\end{array}$ & 1 & 2 & ${ }_{-} I_{-} I_{-}$ \\
\hline 0) & $\begin{array}{l}\text { Varieties of language are a cen- } \\
\text { tral concept in materials. }\end{array}$ & 1 & 2 & $I_{-} I_{-}$ \\
\hline & $\begin{array}{l}\text { Fluency and acceptable lan- } \\
\text { guage are primary goals, and } \\
\text { Bolaccuracy in tenns offonnal } \\
\text { correctness (but rather what is } \\
\text { appropriate for the context) o }\end{array}$ & 1 & 2 & ${ }_{-} I_{-} I_{-}$ \\
\hline
\end{tabular}




\begin{tabular}{|c|c|c|c|c|}
\hline & $\begin{array}{l}\text { Classroom goals are focused on } \\
\text { aH of the components (gram- } \\
\text { matical, discourse, functional, } \\
\text { sociolinguistic, and strategic } \\
\text { components) of communica- } \\
\text { tive competence. }\end{array}$ & 1 & 2 & ${ }_{-} I_{-} I_{-}$ \\
\hline r) & $\begin{array}{l}\text { Teacher allows students lo fo- } \\
\text { cus on Iheir own learning pro- } \\
\text { cess Ihrough: } \\
\text { lhe understanding of their own } \\
\text { learning slyles } \\
\text { the development of appropriate } \\
\text { strategies for self-learning. }\end{array}$ & 1 & 2 & ${ }_{-} I_{-} I_{-}$ \\
\hline S) & $\begin{array}{l}\text { Holistic assessment techniques } \\
\text { in tesling. }\end{array}$ & 1 & 2 & ${ }_{-} I_{-} I_{-}$ \\
\hline t) & $\begin{array}{l}\text { Integration of Ihe four basic } \\
\text { skiHs. }\end{array}$ & 1 & 2 & $I_{-} I_{-}$ \\
\hline
\end{tabular}

